Absorption and diffusion of beryllium in graphite, beryllium carbide formation investigated by density functional theory

Yves Ferro' , Alain Allouche, and Christian Linsmeier

Citation: Journal of Applied Physics 113, 213514 (2013); doi: 10.1063/1.4809552

View online: http://dx.doi.org/10.1063/1.4809552

View Table of Contents: http://aip.scitation.org/toc/jap/113/21

Published by the American Institute of Physics

\section{AIP Journal of ArPplied Physics}

Save your money for your research.

It's now FREE to publish with us no page, color or publication charges apply.
Publish your research in the

Journal of Applied Physics

to claim your place in applied

physics history. 


\title{
Absorption and diffusion of beryllium in graphite, beryllium carbide formation investigated by density functional theory
}

\author{
Yves Ferro, ${ }^{1, a)}$ Alain Allouche, ${ }^{1}$ and Christian Linsmeier ${ }^{2}$ \\ ${ }^{1}$ Laboratoire de Physique des Interactions Ioniques et Moléculaires Aix-Marseille UniversitélCNRS-UMR \\ 7345, Campus de Saint Jérôme, Service 252, 13397 Marseille Cedex 20, France \\ ${ }^{2}$ Max-Planck-Institut für Plasmaphysik, EURATOM Association, Boltzmannstrasse 2, 85748 Garching b. \\ München, Germany
}

(Received 13 February 2013; accepted 20 May 2013; published online 7 June 2013)

\begin{abstract}
The formation of beryllium carbide from beryllium and graphite is here investigated. Using simple models and density functional theory calculations, a mechanism leading to beryllium carbide is proposed; it would be (i) first diffusion of beryllium in graphite, (ii) formation of a metastable beryllium-intercalated graphitic compound, and (iii) phase transition to beryllium carbide. The growth of beryllium carbide is further controlled by defects' formations and diffusion of beryllium through beryllium carbide. Rate limiting steps are the formation of defects in beryllium carbide, with estimated activation energies close to $2 \mathrm{eV}$. (C) 2013 AIP Publishing LLC.
\end{abstract}

[http://dx.doi.org/10.1063/1.4809552]

\section{INTRODUCTION}

One of the key issues for the successful development of the fusion reactor ITER (International Tokamak Experimental Reactor) is the selection of the suitable Plasma Facing Components (PFCs) for the inner wall of the vacuum chamber containing the plasma. PFCs have to fulfill many requirements such as lifetime and safety issues due to tritium retention. In the first stage of ITER operation, PFC will be made of beryllium, tungsten, and possibly carbon in its graphitic form. ${ }^{1}$ A lot of data have already been accumulated about pure carbon, and the current focus is more about tungsten and beryllium. Nevertheless, carbon is still scheduled to be part of the first stage of ITER operation, and future use of carbon in fusion devices cannot be excluded. In addition to pure materials, mixed materials are also expected to form under operating conditions. Erosion and depositions processes activated by thermal load and particle fluxes will favor the formation of these mixed compounds. In particular, beryllium in ITER will be subject to strong physical sputtering. Deposition of beryllium on carbon will lead to mixed Be/C materials to which very little is known at the moment. Experimentally, the interaction of beryllium with carbon surfaces is very difficult to address due to the high reactivity of beryllium with oxygen. However, some clear evidences of beryllium carbide $\mathrm{Be}_{2} \mathrm{C}$ are derived from XPS measurements in Laboratory experiments, in which chemical reaction between beryllium and graphite was shown to proceed from room temperature ${ }^{2,3}$ to $623 \mathrm{~K}$ (Ref. 4) and higher temperature. The first wall and the divertor area of the ITER vacuum vessel will be baked at $515 \mathrm{~K}$ and $623 \mathrm{~K},{ }^{5}$ respectively, in the range of temperature in which beryllium carbide is expected to be formed. The formation of mixed $\mathrm{Be} / \mathrm{C}$ materials was also investigated in the PIESCES-B facility. Formation of mixed Be/C materials was observed and shown to reduce the sputtering yield of pure carbon materials exposed to high plasma flows. These mixed

a)yves.ferro@univ-amu.fr materials were also evidence as beryllium carbide from XPS measurements. ${ }^{6-8}$

From a theoretical point of view, very little is known about the mechanisms of formation of beryllium carbide and its properties. Molecular Dynamic (MD) simulations were already conducted about the sputtering mechanisms of $\mathrm{Be}_{2} \mathrm{C}{ }^{9,10}$ Simulations with the 3D Monte-Carlo code ERO are also reported, with the aim to reproduce the mitigation effect of sputtering properties induced by mixed $\mathrm{Be} / \mathrm{C}$ material. ${ }^{11,12}$ However, the mechanism of formation of $\mathrm{Be}_{2} \mathrm{C}$ remains unknown; it is just parameterized in ERO as a characteristic time $\tau_{\mathrm{B} / \mathrm{C}}$ for $\mathrm{Be}_{2} \mathrm{C}$ formation, which is fitted to reproduce at best the experimental results.

Hence, the present paper is devoted to the understanding of the mechanisms leading to the formation of beryllium carbide. The energy data here computed will be discussed assuming beryllium atoms deposited on the graphite surface with thermal kinetic energy. Nevertheless, if with higher energy, beryllium atoms will be trapped in graphite bilayers and then thermalized. Furthermore, if ionized, beryllium atoms will be neutralized by contact with the $\pi$ system of graphite. As a consequence, only beryllium atoms can be considered to understand the mechanism leading to beryllium carbide.

From a fundamental point of view, the interaction of beryllium atom with the surface of graphite ${ }^{13,14}$ and nanoribbons of graphite ${ }^{15}$ was previously investigated by density functional theory (DFT). Beryllium was shown not to significantly bond on the graphite surface; this is the opposite to what happens in the bulk of graphite, where a tendency to beryllium clustering was established. ${ }^{13}$ In the continuity of this work and also using DFT, the diffusion of beryllium in perfect and defective graphite is here investigated, and the impact of defects on the diffusion process and chemical reactivity of graphite is assessed. An upper bond to activation energies related to these mechanisms is proposed, and mechanisms leading to the growth of $\mathrm{Be}_{2} \mathrm{C}$ at the interface between beryllium and graphite as experimentally observed ${ }^{2-7}$ are established. The focus of the present paper is eventually to provide 
nuclear fusion science with mechanisms that could open the way to comparison with MD calculations, and fundamental data to be integrated in simulation codes based on MonteCarlo or rate-equations techniques.

\section{COMPUTATIONAL DETAILS}

The Quantum Espresso code ${ }^{16}$ developed in a planewaves basis set was used with the ultra-soft pseudo-potentials $^{17}$ and energy cutoffs of $35 \mathrm{Ry}(476 \mathrm{eV})$ and $200 \mathrm{Ry}$ $(2720 \mathrm{eV})$ for the truncation of the plane-wave expansion of the wave function and the electronic density, respectively. In a number of previous works, ${ }^{14,15}$ the interaction of beryllium with graphitic systems was investigated by DFT with the PBE functional. ${ }^{18}$ In line with these authors, and including the van der Waals interaction in graphite according to the work of Barone et al., ${ }^{19}$ all the calculations were performed with the Perdew-Burke-Ernzerhof generalized gradient approximation of the exchange and correlation functional, complemented by the Grimme correction DFT-D2. ${ }^{20}$ The same methodology was carried on in a previous study. ${ }^{13}$ Minimum energy path and the corresponding saddle-points were investigated using the nudged elastic band (NEB) method. ${ }^{21,22}$

The surface and the bulk properties of graphite were modeled by a single graphene layer ( 64 carbon atoms) and a graphite bilayer (128 carbon atoms), respectively. A $4 \times 4 \times 1$ pseudo $2 \mathrm{D}$ supercell in which each image is repeated every $20 \AA$ along the $z$ axis was used as in Refs. 13, 23, and 24. The dimensions of the unit cell were optimized to $\mathbf{a}=\mathbf{b}=2.463 \AA$ and subsequently used in all the calculations. The interlayer distance of the bilayer was set to its experimental value in graphite, i.e., $3.35 \AA$. All the atoms were allowed to relax during the geometry optimizations, except those at the border of the working-cell in order to simulate the constraints imposed by the infinite layer(s) on the cell. The Brillouin zone related to the $4 \times 4 \times 1$ supercell was sampled in a $6 \times 6 \times 1$ mesh using the Monkhorst-Pack scheme, ${ }^{25}$ which is equivalent to a $24 \times 24 \times 1$ mesh in the unit cell. Other carbon structures are further investigated, such as nanoribbons and beryllium carbide; they will be described later in the corresponding sections.

\section{RESULTS}

It was theoretically shown that beryllium does not react at the surface of perfect graphite; it just physisorbs on it, with adsorption energies of about a hundred meV. ${ }^{13,15,26,27}$ Thus, in case of beryllium evaporation and redeposition on graphite, the first step leading to beryllium carbide would be beryllium diffusion in graphite, then beryllium clustering, followed by a phase transition. As a consequence, we first start to investigate different models for beryllium diffusion into perfect and defective graphite.

\section{A. Diffusion of Be in perfect graphite}

A beryllium atom coming from the gas phase was first considered. In that case, beryllium has to penetrate into graphite by crossing the first graphene layer of the surface. Then, once in graphite, beryllium has to diffuse into the bulk.

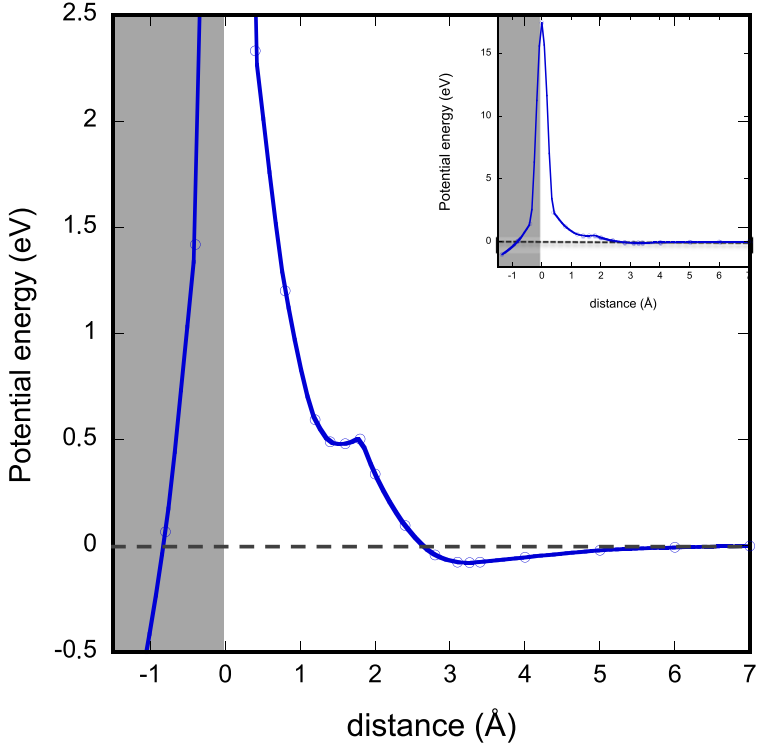

FIG. 1. Potential energy surface of a Be atom from infinity crossing the layer surface of the graphite bilayer at $d=0 \AA$. The interior of the bilayer is shown in gray. The full curve is given in inset.

\section{Across the graphene layers}

Figure 1 displays the potential energy curve of a beryllium atom from the gas phase crossing the first graphene layer; the surface is at the origin of the distance axis. A $87 \mathrm{meV}$ depth physisorption well was found at $3.120 \AA$ from the surface. This result confirms the weak interaction of beryllium with the surface of graphite. The main characteristic of that curve is a huge activation barrier at $17.5 \mathrm{eV}$ when beryllium lies in the graphitic plane. Such a barrier would prevent any diffusion mechanism to happen within the range of temperature considered here (from room temperature to $623 \mathrm{~K})$. Of course, such a result was highly predictable, since it was shown experimentally ${ }^{28}$ and confirmed theoretically ${ }^{29}$ that helium is not able to diffuse that way, as even was shown for the small hydrogen atoms. ${ }^{30}$ However, once in the bilayer, beryllium chemisorbs with an energy of $\mathrm{E}_{\mathrm{ads}}=-1.03 \mathrm{eV}$, at $1.56 \AA$ below the surface.

\section{In between the graphene layers}

The minimum energy path connecting two chemisorption sites in the bilayer was scanned. In the chemisorption well, beryllium is on top of a carbon atom and below a carbon ring. The activation energy to the first nearest chemisorption site is $E_{a}=149 \mathrm{meV}$ as shown in Figure 2(a). It is $\mathrm{E}_{\mathrm{a}}=68 \mathrm{meV}$ to the second nearest chemisorption site (Figure 2(b)), which constitute the true minimum energy path. This weak activation energy makes diffusion a very easy process in the range of temperature considered here.

\section{B. Diffusion of Be in defective graphite}

Since diffusion of beryllium across the graphene layers is impossible, a route for beryllium to penetrate inside the bulk of graphite is to be found. The impact of defects on the diffusion process was then investigated, and two different types of local and extended defects were modeled: the 

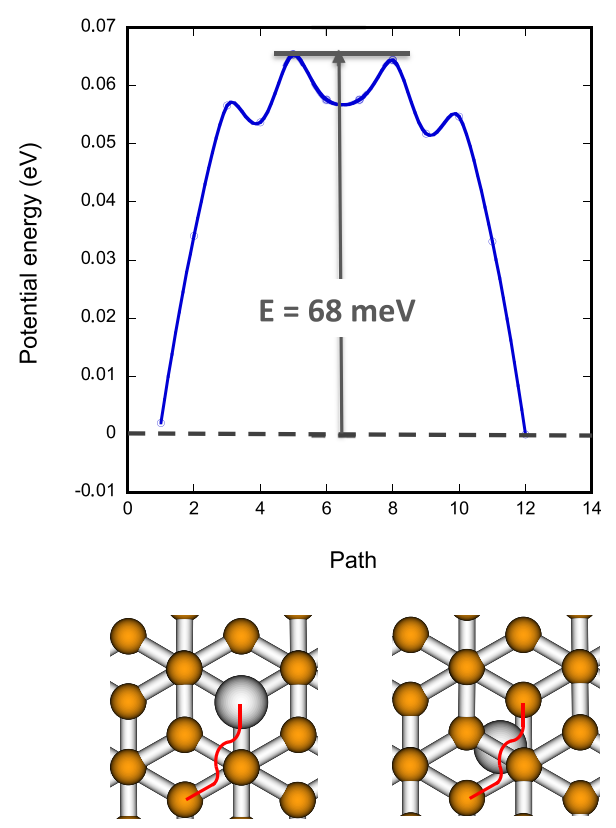

(a)

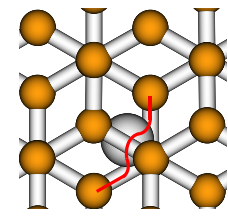

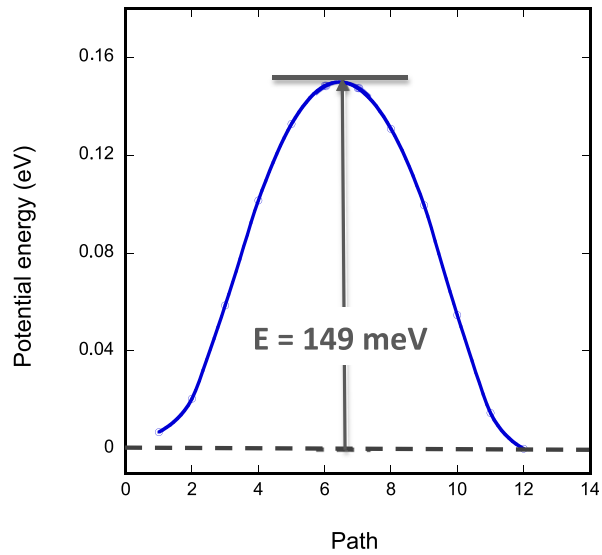
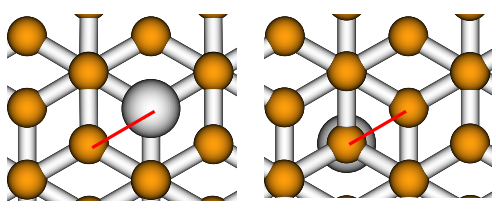

FIG. 2. Minimum energy path (shown in red) between two adsorption sites for beryllium in a bilayer of graphite: (a) Path to join the two first nearest neighbors' sites; initial and final positions are shown below the graph. (b) Path to join the second nearest neighbors' sites; initial and intermediate positions are shown below the graph.

(b)

atomic vacancy and the zigzag and armchair edges of graphite.

\section{The atomic vacancy}

a. The model. The atomic vacancy is the simplest model defect in graphite and as a consequence was extensively investigated by DFT; ${ }^{31,32}$ it was obtained by just removing one carbon atom from the bilayer with a subsequent geometry relaxation. Several electronic and magnetic configurations were found, each characterized by different spin states. In order to select the electronic ground states, different spin configurations were investigated: singlet $(S=0)$, triplet $(S=1)$, quintet $(S=2)$, and relaxed spin.

In the case of the singlet spin state, the symmetry is broken in order to allow for antiferromagnetic states with nonzero absolute magnetization; however, no antiferromagnetic configuration was found. The genuine electronic ground state is triplet $(\mathrm{S}=1)$ as shown in Table I. Both singlet and quintet electronic states are $0.129 \mathrm{eV}$ and $1.263 \mathrm{eV}$ higher in energy, respectively, and the relaxed spin case converged to $\mathrm{S}=1$. As a consequence, only the triplet is considered in the following. The atomic vacancy defect is, therefore, ferromagnetic, which is consistent with experimental observations. ${ }^{33}$

b. Adsorption near the atomic vacancy. The reactivity of the vacancy toward beryllium has first to be investigated. Four different adsorption within the bilayer sites were

TABLE I. Atomic vacancies with $\mathrm{S}=0,1$, and 2. $\Delta \mathrm{E}$ from GS: difference in energy from the ground state, $\mathrm{S}=1$.

\begin{tabular}{cccc}
\hline \hline & $\begin{array}{c}\Delta \mathrm{E} \text { from } \\
\text { GS }(\mathrm{meV})\end{array}$ & $\begin{array}{c}\text { Total } \\
\text { magnetization }\left(\mu_{\mathrm{B}}\right)\end{array}$ & $\begin{array}{c}\text { Absolute } \\
\text { magnetization }\left(\mu_{\mathrm{B}}\right)\end{array}$ \\
\hline $\mathrm{S}=0$ & 129 & 0 & 0 \\
$\mathrm{~S}=1$ & 0 & 2 & 3 \\
$\mathrm{~S}=2$ & 1263 & 4 & 4.6 \\
\hline \hline
\end{tabular}

selected close to the vacancy as shown in Figure 3. The equilibrium geometries were obtained starting from the geometry of adsorption in the perfect bilayer and then relaxed. The corresponding adsorption energies are given in Table II. Two sets of results can be drawn. Sites 1 and 2 exhibit the lowest adsorption energies and consequently the highest stability. They belong to the nearest carbon rings close to the vacancy. There, borders effects are expected to be most sensitive $^{34}$ and to induce a high reactivity. ${ }^{35}$ Positions 3 and 4 belong to the second nearest carbon ring. While border effects mitigate, the chemisorption well at the adsorption sites remains above $0.5 \mathrm{eV}$ below that in the perfect bilayer.

c. Diffusion through the atomic vacancy. The minimum energy paths for atomic beryllium from the gas phase to sites 1 and 2 are shown in Figure 4. Distances are given from the coordinate of the missing carbon atom at the center

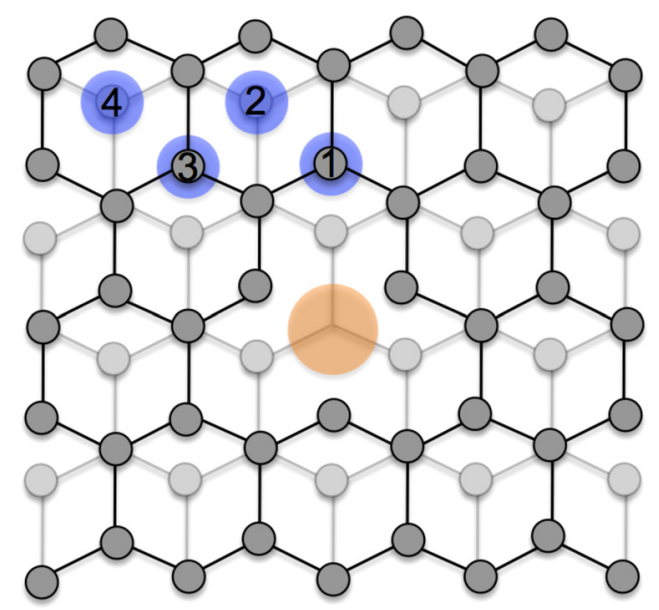

FIG. 3. Atomic vacancy in the upper layer of the bilayer modeling the surface. Sites 1 to 4 are minimum energy geometries for Be in between the bilayer and close to the vacancy. Sites 1 and 3 are below a carbon atom of the upper layer and sites 2 and 4 are above a carbon of the lower layer. 
TABLE II. Energy of adsorption of a Be atom in a bilayer of graphite close to an atomic vacancy. Sites are labeled according to Figure 3. Adsorption in a perfect bilayer is also given as a reference.

\begin{tabular}{lc}
\hline \hline Site & $\mathrm{E}_{\mathrm{ads}}(\mathrm{eV})$ \\
\hline 1 & -1.77 \\
2 & -1.78 \\
3 & -1.51 \\
4 & -1.55 \\
Bilayer & -1.02 \\
\hline \hline
\end{tabular}

of the atomic vacancy. At a beryllium-surface distance of about $1 \AA$ above, the surface distorts toward vacuum, with a corresponding deep minimum in energy $\left(\mathrm{E}_{\mathrm{ads}}=-6.09 \mathrm{eV}\right)$. Another minimum is observed, corresponding to a symmetric distortion toward the interior of the bilayer. This double well indicates the possibility of a flip-flop for a Be atom stuck in that potential well. The main characteristic of both those curves leading to sites 1 and 2 is, however, the absence of any activation barrier. Assuming an elastic process, the beryllium diffusing through an atomic vacancy from the gas phase is trapped within the bilayer, carrying $1.77 \mathrm{eV}$ of kinetic energy. In the case of an inelastic process, beryllium would loose its energy in the graphene lattice and could be trapped in the vacancy. This point will be discussed later on.

\section{Zigzag and armchair edges}

a. The model. Modeling a zigzag or armchair edge involves $\sigma$ bonds' breaking. This induces some dramatic modifications in the electronic structure of the graphene layers, along with a dramatic increase in chemical reactivity. Hydrogen is the prominent residual gas in low-pressure experiments. Of course, other molecules are present in the

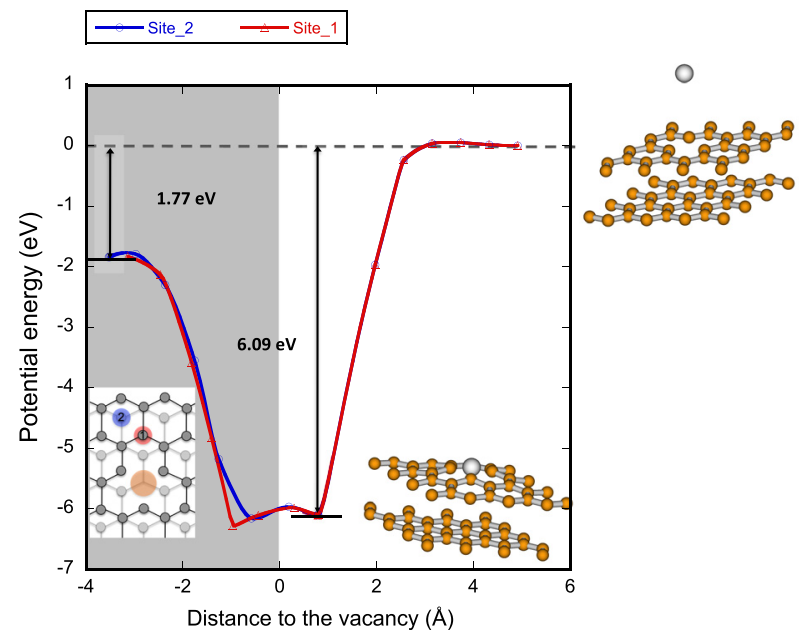

FIG. 4. Diffusion of a beryllium atom across an atomic vacancy up to sites 1 and 2 inside the bilayer. Distances are given with respect to the missing carbon atom; positive values are toward vacuum, negatives values are inside the bilayer (shown in grey). The molecular models display from the right to the center of the figure: the beryllium atom $5 \AA$ and $1 \AA$ above the surface (minimum in energy), respectively. The final absorption sites 1 and 2 are shown on the left in the inset. experimental conditions and can react with graphite. ${ }^{36,37}$ Their concentrations are, however, significantly lower; therefore, it can be considered that saturation of the dangling $\sigma$ bonds by $\mathrm{H}$ atoms is an accurate representation of the experimental conditions. As shown in Figure 5, four nanoribbons were piled-up with the same Bernal stacking as in graphite. The cell dimensions are $19.0 \times 8.532 \times 20.0 \AA$ for the $\mathrm{arm}$ chair and $19.0 \times 9.852 \times 20.0 \AA$ for the zigzag edge. The unit cell contains 160 atoms in both cases. The Brillouin zone was sampled using a $1 \times 6 \times 1$ mesh.

Cutting an armchair edge in graphene does not produce any perturbation in the $\pi$ system, on the contrary to the zigzag edge where border effects are introduced. ${ }^{38-40}$ Scanning tunneling microscopy is an efficient tool to reveal these features. ${ }^{41-43}$ Borders effects have an exponential decay when going towards the bulk material. ${ }^{39}$ In addition to border effects, single-layers zigzag nanoribbons were calculated to produce antiferromagnetic states, ${ }^{44}$ nevertheless, these electronic states have never been observed experimentally, and they are predicted to vanish at room temperature. ${ }^{45}$ Anyway, the magnetic states disappear when zigzag nanoribbons are piled up as in the present case, as indicated also by Lee et $a l .^{46}$ The non-magnetic state was confirmed in the present case according to the following way: the symmetry of the system was broken in order to allow for brokensymmetry solutions, and the starting magnetization was set to antiferromagnetic at the beginning of the calculation. The calculation ran with no constraint on spin finally converged to a non-magnetic electronic ground state.

b. Diffusion. Figure 6 displays the potential energy surface of a beryllium atom coming from the gas phase and penetrating both the armchair and zigzag edges in between the second and third layers of the model. The paths are shown in Figures 5(b) and 5(c), respectively. Here again, these processes occur without any activation barrier; the mechanisms leading to the center of the nanoribbons are exothermic with gains in energy of $1.64 \mathrm{eV}$ and $2.16 \mathrm{eV}$ for the armchair and zigzag edges, respectively. The adsorption energy at the border of the zigzag edge is of $-3.47 \mathrm{eV}$; it is $-1.86 \mathrm{eV}$ at the border of the armchair edge, which confirms the higher reactivity of the zigzag edge. Border effects tend to vanish when penetrating deeper into the bilayer; the adsorption energy should tend to $\mathrm{E}_{\mathrm{ads}}=-1.03 \mathrm{eV}$ as in perfect graphite. However, such mitigation is a long distance process, which should be modeled by such a large system that it would be out of our computing capabilities. As a consequence, the adsorption energies remain well below $-1.03 \mathrm{eV}$ at the center of both armchair and zigzag nanoribbons.

Finally, the main conclusion of this paragraph is that both the atomic vacancy and the zigzag and armchair edges display similar energy profiles characterized by zero activation barriers. As a consequence, assuming elastic processes, these defects would promote beryllium diffusion into the bulk of graphite. Otherwise, in cases where beryllium would loose enough energy to get stuck into the defect, beryllium would accumulate in the vicinity of the defects, which would probably be the starting point for beryllium carbide formation as will be see later. 


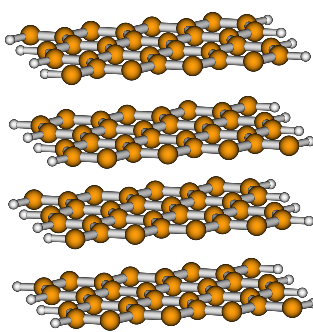

(a)

\section{The beryllium carbide formation}

The following points were established up to now:

- beryllium penetrates freely into graphite through surface defects,

- beryllium diffuses in between the graphene layers with very low activation barriers.

Moreover, the tendency of beryllium to aggregate into planar clusters in between the graphene bilayers was previously established. ${ }^{13}$ In the following, it is assumed that the subsequent diffusion and aggregation processes lead to the formation of complete layers of beryllium in the graphene bilayer.

\section{Lamellar Be/C structure}

As a first step, a complete beryllium layer was built in the graphene bilayer (Figure 7(a)). Such intercalated compounds were already observed with calcium as $\mathrm{CaC}_{6} ;{ }^{47,48}$ these compounds have potential high hydrogen storage capacity $^{49}$ and superconducting properties. ${ }^{50}$ Berylliumintercalated graphitic compounds $\mathrm{BeC}_{6}$ were also investigated
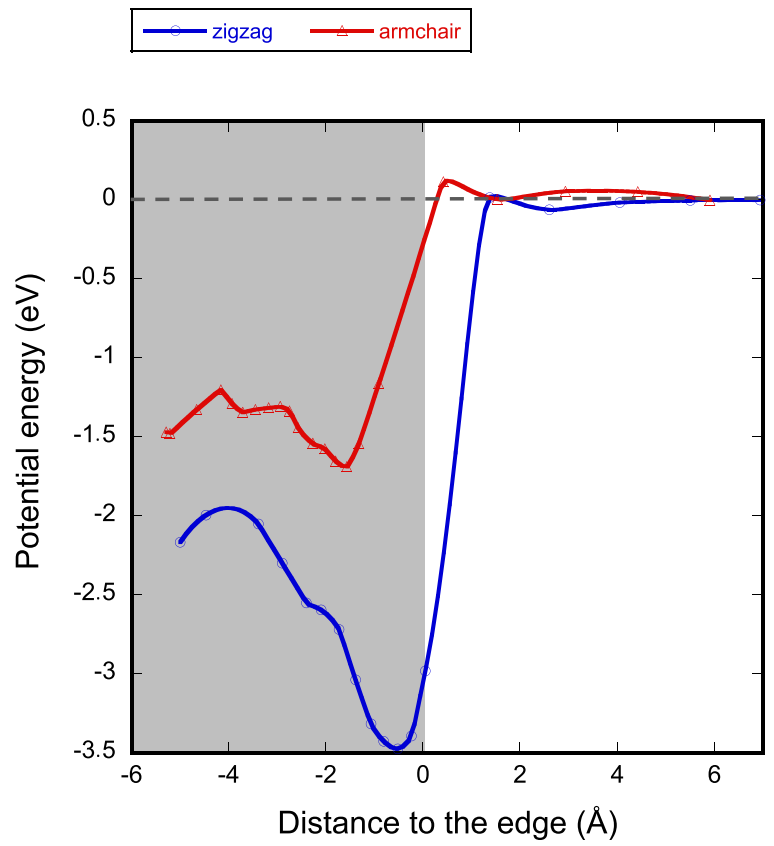

FIG. 6. Potential energy surface of a Be atom from vacuum penetrating the center of the nano-ribbons. The origin of the $x$ axis is on the edge (coordinates of the H-terminating atoms). The shaded area represents the bulk material.

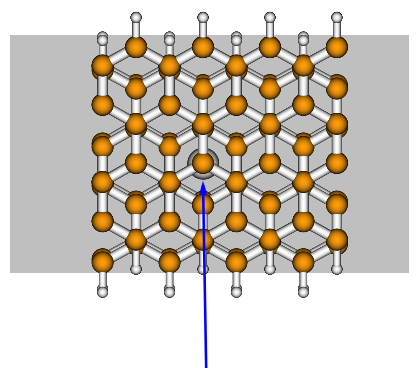

FIG. 5. (a) The 4 nano-ribbons model containing 160 carbon atoms modeling the edge of graphite. (b) Armchair edge with a Be atom at the center of the nanoribbons. The path further investigated by the NEB technique is shown by an arrow. (c) Zigzag edge with a Be atom at the center of the nano-ribbons. The NEB path is shown by an arrow. as hydrogen storage materials. ${ }^{51}$ In the beryllium-intercalated graphitic compound represented in Figure 7(a), beryllium atoms are on top of a carbon ring and below a carbon atom (or vice versa), arranged in a hexagonal structure in its equilibrium geometry. In that case, the unit cell contains 64 carbon atoms and 32 beryllium atoms. The formation energy per $B e$ atom is calculated according to the following equation:

$$
\Delta E_{B e_{n}}=\frac{1}{N}\left(E_{B e_{n} C_{m}}-E_{\text {bilayer }}-N E_{B e}\right),
$$

where $\mathrm{N}$ stands for the number of beryllium atoms in the unit cell (here, $N=32$ ). The formation energy of $\mathrm{Be}_{2}, \mathrm{Be}_{3}$, $\mathrm{Be}_{6}$, and $\mathrm{Be}_{7}$ clusters in a bilayer of graphite has been evaluated in a previous work: ${ }^{13} \Delta \mathrm{E}_{\mathrm{Be} 2}=-1.042 \mathrm{eV} / \mathrm{Be}$ atom, $\Delta \mathrm{E}_{\mathrm{Be} 3}=-1.306 \mathrm{eV} / \mathrm{Be}$ atom, $\Delta \mathrm{E}_{\mathrm{Be} 6}=-1.508 \mathrm{eV} / \mathrm{Be}$ atom, and $\Delta \mathrm{E}_{\mathrm{Be} 7}=-1.653 \mathrm{eV} / \mathrm{Be}$ atom. As shown in Table III, $\Delta \mathrm{E}_{\mathrm{BeC} 2}=-2.283 \mathrm{eV} / \mathrm{Be}$ atom. These results emphasize the capability of beryllium atoms to aggregate into planar structures up to completion of a beryllium layer.

The concentration in beryllium atoms was further increased by building successive layers of beryllium inside the bilayer of graphite. The corresponding stoichiometries are $\mathrm{BeC}, \mathrm{Be}_{3} \mathrm{C}_{2}, \mathrm{Be}_{2} \mathrm{C}$, and $\mathrm{Be}_{3} \mathrm{C}$. Inside the carbon bilayer, beryllium atoms packed together according to the same hexagonal structure as in bulk beryllium. An example of such a structure is given in Figure 7(b) in the case of $\mathrm{Be}_{2} \mathrm{C}$ (four layers of beryllium atoms packed in hexagonal structure). The corresponding formation energies per beryllium atom as defined in Eq. (1) are given in Table III for all the compounds. High beryllium concentration compounds are favored and the

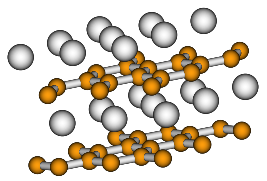

(a)

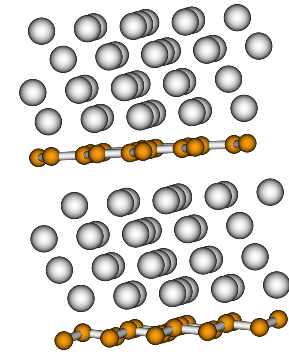

(b)

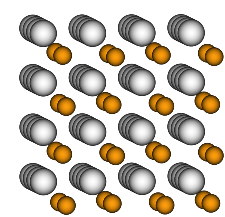

(c)
FIG. 7. (a) Intercalated $\mathrm{BeC}_{2}$ compound. $\mathrm{Be}$ are at the minimum energy position found for a single Be atom in between a bilayer of graphite: on top of a $\mathrm{C}$ atom (lower layer) and in the middle of a carbon ring (upper layer). (b) Intercalated $\mathrm{Be}_{2} \mathrm{C}$ compound. Starting from the configuration of $\mathrm{BeC}_{2}$, Be atoms are arranged in an hcp configuration. (c) Beryllium carbide in the thermodynamic stable $f c c$ structure. 
TABLE III. Energy of interaction per Be atom. Reference compounds are atomic beryllium and $\mathrm{C}$ (graphite). In all compounds, berylliums atoms are intercalated in the graphitic structure, except reference compound Be bulk and $\mathrm{Be}_{2} \mathrm{C}(f c c)$.

Compound $\quad \mathrm{BeC}_{2} \quad \mathrm{BeC} \quad \mathrm{Be}_{3} \mathrm{C}_{2} \quad \mathrm{Be}_{2} \mathrm{C} \quad \mathrm{Be}_{3} \mathrm{C} \quad$ Be bulk $\quad \mathrm{Be}_{2} \mathrm{C} f c c$

Energy (eV) $-2.283 \quad-3.148 \quad-3.255 \quad-3.273 \quad-3.493 \quad-3.880 \quad-4.390$

formation energy per Be atoms tends towards the limit of bulk beryllium. As expected, the formation energy per Be atoms tends to the limit of bulk beryllium. However, a most stable compound exists with $\mathrm{Be}_{2} \mathrm{C}$ stoichiometry, which is known as beryllium carbide and crystallized according to a face centered cubic structure $f c c$ (Figure $7(\mathrm{c})$ ). The gain in energy from $\mathrm{Be}_{2} \mathrm{C}$ in hexagonal symmetry $(-3.148 \mathrm{eV} / \mathrm{Be}$ atom-metastable) to $\mathrm{Be}_{2} \mathrm{C}$ in $f c c$ structure $(-4.390 \mathrm{eV} / \mathrm{Be}$ atom thermodynamically stable) is $1.242 \mathrm{eV} / \mathrm{Be}$ atom.

As a consequence, once incorporated into graphite, beryllium tends to form intercalated compounds, before a phase transition toward the thermodynamically stable structure of beryllium carbide takes place.

\section{A proposed mechanism to beryllium carbide}

The mechanism leading to the $f c c$ beryllium carbide and the related activation energy are addressed in the present paragraph. Of course, a complete answer should only be given by considering the dynamics of the system, which is out of the scope of the present work, and would not be possible within the DFT framework on such large structures. However, a plausible mechanism can be proposed, which allows providing an upper limit to the activation energy required for the phase transition from the intercalated compound to $f c c \mathrm{Be}_{2} \mathrm{C}$. The prerequisites for such a mechanism are as follows:

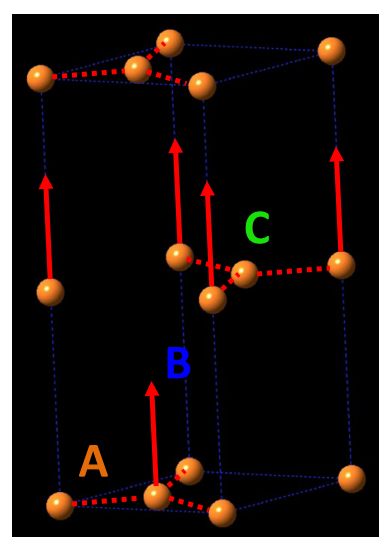

(a)

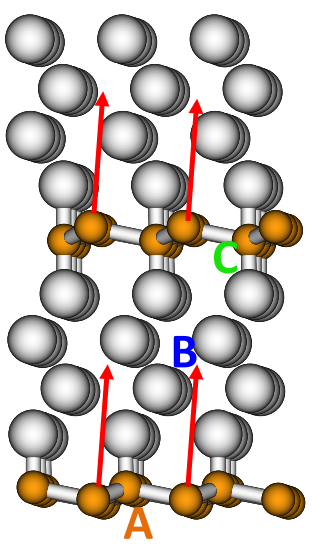

(b)
FIG. 8. Distortion of the carbon atom network from the $A B A$ hexagonal structure (shown in (a) and $(\mathrm{b}()$ to the $A B C$ staking of the $f c c$ beryllium carbide. One of two carbon atoms is moved in each layers of the initial hexagonal structure. In the final cubic structure, the A layer is formed by the carbon atoms left on the bottom layer, the B layer is generated by moving one of two carbon atoms from the bottom layer to the intermediate space of the bilayer, the $\mathrm{C}$ layer contains the carbon atoms left on the intermediate layer. (a) Graphite unit cell only, for more clarity and (b) full structure with beryllium atoms.
- the beryllium structure is metallic and, therefore, fairly soft and can easily accommodate distortions of its environment,

- the carbon structure (graphene in its initial stage) is much harder. As phase transition to a $f c c$ structure requires $\mathrm{C}-\mathrm{C}$ bond breaking, the activation energy is likely to be driven by the carbon network distortion on which we focused in the following.

Carbon layers pile up according to an $A B$ stacking sequence in a hexagonal symmetry. In an $f c c$ structure, the carbon stacking is $A B C$ according to the diagonal of the cubic cell. The hexagonal structure is characterized by a cell parameter $\mathbf{a}=2.463 \AA$ and an interlayer distance of $12.453 \AA$ when filled with beryllium. The $f c c$ structure is characterized by a cell parameter $\mathbf{a}=4.310 \AA\left(\mathbf{a}_{\exp }=4.342 \AA\right)$ and an interlayer distance of $2.489 \AA$.

As a consequence, the model was set up as follows:

- the working cell is a $1 \times 1 \times 3$ supercell containing two carbon atoms per layer and six carbon layers along the $\mathrm{z}$ axis (three $A B$ units),

- this unit cell is filled with beryllium atoms in an hexagonal packing to achieve the $\mathrm{Be}_{2} \mathrm{C}$ stoichiometry,

- the carbon atoms are progressively moved from an $A B$ stacking to an $A B C$ stacking as shown in Figure 8. The final cubic structure is consistent with a 12 carbon layers model (four $A B C$ units).

This model allows us to build periodic cubic and hexagonal structures with the same number of carbon and beryllium atoms. The hexagonal structure consists of three unitcells and the cubic structure comprises four unit cells. The cell parameters are linearly fitted from the hexagonal to the cubic structure.

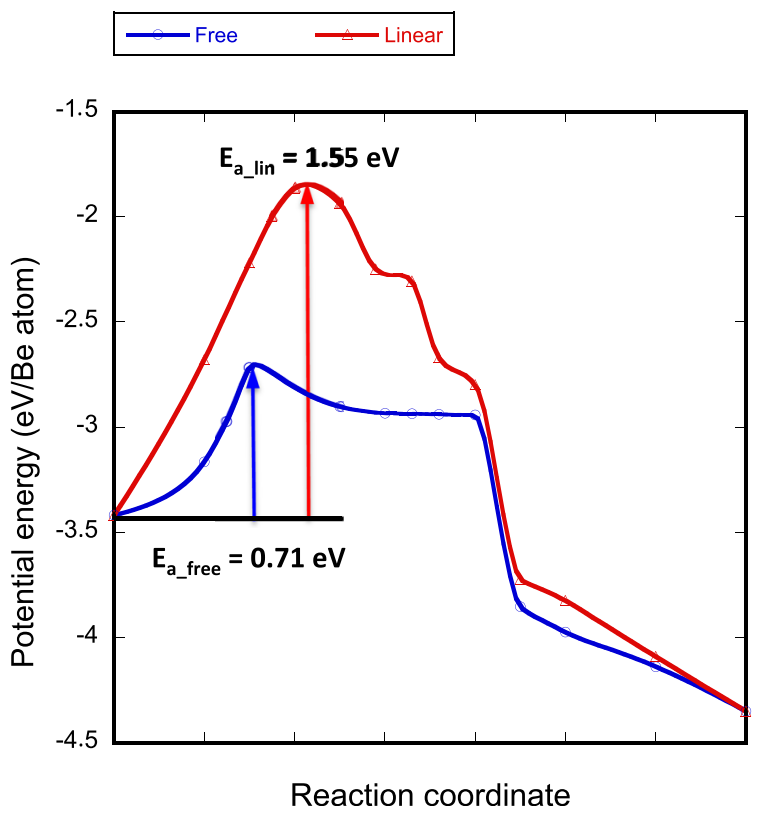

FIG. 9. Potential energy curve of the phase transition from the graphitic hexagonal structure of the lamellar $\mathrm{Be}_{2} \mathrm{C}$ compound to the $f c c$ structure of beryllium carbide. In red and triangle: the cell parameters are linearly scaled from the hexagonal structure to the cubic one. In blue and round, the $c$ parameter is left free to relax. The reference energy is graphite for carbon and atomic beryllium. The energy of interaction is given in $\mathrm{eV}$ per $\mathrm{Be}$ atom. 

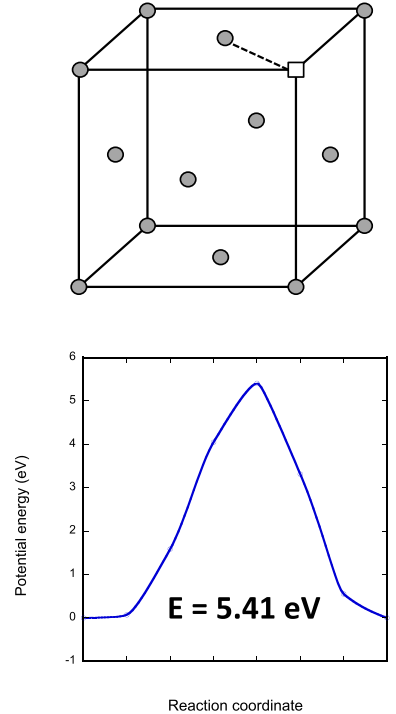

(a)
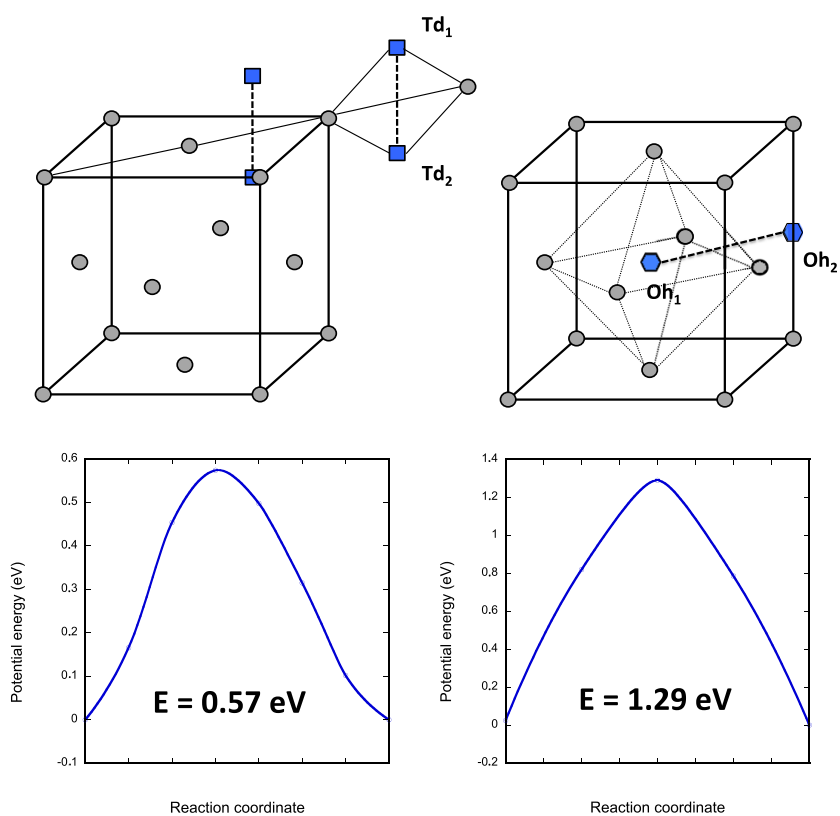

(b)

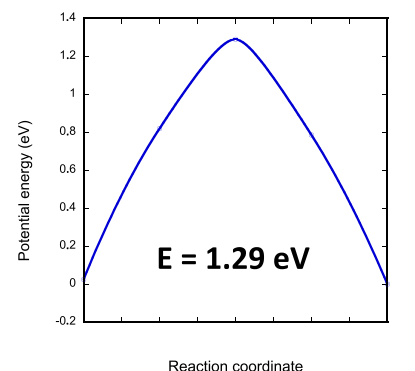

(c)
FIG. 10. Diffusion in $f c c$ beryllium carbide. For more clarity, only the carbon atoms of the $f c c$ structure are displayed. Beryllium atoms are located in all the tetrahedral sites (Td). Paths and energy profiles of (a) a carbon vacancy, (b) a beryllium vacancy from a tetrahedral site $\left(\mathrm{Td}_{1}\right)$ to its next nearest neighbor tetrahedral site $\left(\mathrm{Td}_{2}\right)$, and (c) an interstitial Be atom diffusing from an octahedral $\left(\mathrm{Oh}_{1}\right)$ site to its next nearest neighbor $\left(\mathrm{Oh}_{2}\right)$.

The resulting potential energy surface is shown in Figure 9 (red curve). The activation energy of the phase transition is $1.55 \mathrm{eV}$. Of course, this is an upper limit to the actual activation energy, since all the carbon atoms are not expected to move altogether, and the cell parameters are not expected to move linearly.

We investigated another relaxation scheme: we relaxed the $c$ parameter of the supercell. The resulting curve is shown in blue in Figure 9. The corresponding activation energy is lower than previously: $0.71 \mathrm{eV}$; however, it is still likely to remain an upper limit to the actual activation energy.

\section{Diffusion of atoms and vacancies in beryllium carbide}

We here consider an interface between bulk beryllium and graphite. Beryllium atoms spontaneously diffuse through graphite defects to accumulate and form a berylliumintercalated graphitic compound. Then, a phase transition takes place leading to beryllium carbide. In this scenario, a

beryllium carbide film fills the interface between graphite and beryllium, so that any further growth of that film would be controlled by transport properties throughout beryllium carbide. The transport properties here investigated are summarized in Figure 10. Diffusion of carbon through a carbon vacancy involves $E_{a}=5.41 \mathrm{eV}$ (Fig. 10(a)), which is too high an energy to happen in the range of temperature considered here. On the contrary, moving a beryllium atom through beryllium vacancies in tetrahedral (Td) sites or through interstitial octahedral $(\mathrm{Oh})$ sites requires much smaller activation energies: $0.57 \mathrm{eV}$ and $1.29 \mathrm{eV}$, respectively. It follows that transport properties through beryllium carbide are driven by beryllium diffusion.

Table IV reports the formation energies of defects in graphite and beryllium carbide. Two formation energies are lower than the other

- the formation energy of a beryllium vacancy in $\mathrm{Be}_{2} \mathrm{C}$ : $\Delta \mathrm{E}_{\mathrm{f}}=2.13 \mathrm{eV}$ with bulk beryllium as a reference,

TABLE IV. Thermodynamic data. Related to graphite: (i) the cohesive energy per carbon atom of a graphite bilayer containing 64 atoms, (ii) the energy of formation in $\mathrm{eV}$ of an atomic vacancy in the graphite bilayer, and (iii) the gain in energy for the substitution of a $\mathrm{C}$ vacancy in the bilayer by a Be atom. Related to beryllium carbide: (i) energy of formation of a beryllium vacancy in beryllium carbide with bulk $\mathrm{Be}$ and $\mathrm{Be}_{2} \mathrm{C}$ as a reference, (ii) energy of formation of a carbon vacancy in beryllium carbide, and (iii) energy of formation of an interstitial beryllium atom in beryllium carbide with respect to two different references: from bulk $\mathrm{Be}_{2} \mathrm{C}$ and graphite, and from bulk $\mathrm{Be}_{2} \mathrm{C}$ and atomic carbon.

\begin{tabular}{|c|c|c|}
\hline & Energy $(\mathrm{eV})$ & Reference formula \\
\hline \multicolumn{3}{|l|}{ Graphite } \\
\hline $\mathrm{E}_{\text {cohesive }}$ & -7.90 & $\mathrm{E}_{\text {cohesive }}=\mathrm{E}_{\text {bilayer }} / 64-\mathrm{E}_{\mathrm{C} \text { atomic }}$ \\
\hline$\Delta \mathrm{E}_{\mathrm{f} \text { vacancy }}$ & 16.48 & $\Delta \mathrm{E}_{\mathrm{f} \text { vacancy }}=\mathrm{E}_{\text {vacancy }}+\mathrm{E}_{\mathrm{C} \text { atomic }}-\mathrm{E}_{\text {bilayer }}$ \\
\hline \multirow[t]{2}{*}{$\Delta \mathrm{E}_{\text {sub Be/vacancy }}$} & -6.09 & 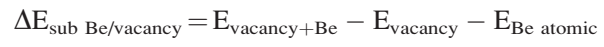 \\
\hline & -2.34 & $\Delta \mathrm{E}_{\text {sub Be/vacancy }}=\mathrm{E}_{\text {vacancy }}+\mathrm{Be}-\mathrm{E}_{\mathrm{vacancy}}-\mathrm{E}_{\mathrm{Be} \text { bulk }}$ \\
\hline \multicolumn{3}{|l|}{ Beryllium carbide } \\
\hline$\Delta \mathrm{E}_{\mathrm{f} \text { Be vacancy }}$ & 2.13 & $\mathrm{E}_{\mathrm{Be} \text { vacancy }}=\mathrm{E}_{\mathrm{Be} \text { vacancy }}-\left(\mathrm{E}_{\mathrm{Be} 2 \mathrm{C}}-\mathrm{E}_{\mathrm{Be} \text { bulk }}\right)$ \\
\hline$\Delta \mathrm{E}_{\mathrm{f}} \mathrm{C}$ vacancy & 4.93 & $E_{C \text { vacancy }}=E_{B e}$ vacancy $-\left(E_{B e 2 C}-E_{C \text { graphite }}\right)$ \\
\hline \multirow[t]{2}{*}{$\Delta \mathrm{E}_{\mathrm{f} \text { Be interstitial }}$} & 6.35 & $\mathrm{E}_{\mathrm{Be} \text { interstitial }}=\mathrm{E}_{\mathrm{Be} 2 \mathrm{C}+\mathrm{Be}}-\left(\mathrm{E}_{\mathrm{Be} 2 \mathrm{C}}+\mathrm{E}_{\mathrm{Be} \text { bulk }}\right)$ \\
\hline & 2.60 & $\mathrm{E}_{\mathrm{Be} \text { interstitial }}=\mathrm{E}_{\mathrm{Be} 2 \mathrm{C}+\mathrm{Be}}-\left(\mathrm{E}_{\mathrm{Be} 2 \mathrm{C}}+\mathrm{E}_{\mathrm{Be} \text { atomic }}\right)$ \\
\hline
\end{tabular}


- the formation energy of an interstitial Be atom from the gas phase, $\Delta \mathrm{E}_{\mathrm{f}}=2.60 \mathrm{eV}$.

We conclude that these are the two mechanisms likely to lead to $\mathrm{Be}_{2} \mathrm{C}$ formation.

\section{DISCUSSION AND CONCLUSION}

In this study, we conclude that high concentrations of beryllium in graphite lead to the formation of beryllium carbide. Beryllium carbide can be formed either by direct interaction of vaporized/atomic beryllium with graphitic compounds, or by deposition as bulk beryllium on top of graphite and then diffusion. We have established a mechanism leading to beryllium carbide from beryllium and graphite. The most important results are summarized below:

- beryllium diffuses spontaneously through defects in graphite,

- beryllium diffuses with a very low activation $\left(\mathrm{E}_{\mathrm{a}} \approx 100 \mathrm{meV}\right)$ energy between the layers of graphite,

- beryllium has a tendency to accumulate and to form intercalated compounds in graphite,

- phase transition from an intercalated compound to beryllium carbide happens with an activation energy below $0.77 \mathrm{eV}$,

- transport properties in $\mathrm{Be}_{2} \mathrm{C}$ are driven by beryllium diffusion through vacancies $\left(\mathrm{E}_{\mathrm{a}}=0.57 \mathrm{eV}\right)$ or interstitials $\left(\mathrm{E}_{\mathrm{a}}=1.29 \mathrm{eV}\right)$,

- the formation energies of beryllium vacancies and interstitials in $\mathrm{Be}_{2} \mathrm{C}$ are (i) $\Delta \mathrm{E}_{\mathrm{f}}=2.13 \mathrm{eV}$ for vacancies formation at the $\mathrm{Be} /$ graphite interface and (ii) $\Delta \mathrm{E}_{\mathrm{f}}=2.60 \mathrm{eV}$ for the formation of interstitial with Be from the gas phase.

The kinetics of the last step (defect formation) was not investigated; defects have to be created at the interface between beryllium carbide and graphite or beryllium. This is a too complex system, whose size brings the models out of the capabilities of DFT methods. It would probably require dynamical methods to be well accounted.

However, all the activation energies involving beryllium are quite low as exemplified in the present paper. As a consequence, the formation energies we get for vacancies formation and defects creation are probably not that far from the activation energies driving the kinetics of the processes; they are at least lower limit values.

Within this approximation, we can conclude that the kinetic of the beryllium carbide growth is controlled by transport properties and creation of defects. If beryllium carbide is formed at the interface between bulk beryllium and graphite, the rate-limiting step is the formation of beryllium vacancies in $\mathrm{Be}_{2} \mathrm{C}$ with activation energies of at least $2.13 \mathrm{eV}$. Then, beryllium is able to diffuse enabling the $\mathrm{Be}_{2} \mathrm{C}$ phase to grow. If it is formed from vaporized beryllium, the ratelimiting step is the creation of interstitial beryllium atoms in $\mathrm{Be}_{2} \mathrm{C}$ with an activation energy of at least $2.60 \mathrm{eV}$.

As a conclusion, mechanisms, thermodynamic and kinetic data were produced in this work to understand the formation of beryllium carbide as experimentally observed. ${ }^{2-8}$ Beryllium carbide is one of the mixed materials likely to form in tokamak conditions. These data are to be used and compared with experiments; they are mainly to be used and integrated in Kinetic Monte-Carlo, rate equations, and any other codes allowing a multiscale approach to the modeling of the walls of tokamaks.

\section{ACKNOWLEDGMENTS}

This work, supported by the European Communities under the partnering contract with EURATOM-CEA, and with the French Research Federation for fusion studies, was carried out within the framework of the European Fusion Development Agreement. The views and opinions expressed herein do not necessarily reflect those of the European Commission. The authors of this work were granted access to the HPC resources of [CCRT/CINES/IDRIS] under the allocation i2012096612 made by GENCI (Grand Equipement National de Calcul Intensif).

${ }^{1}$ J. Roth, E. Tsitrone, A. Loarte, T. Loarer, G. Counsell, R. Neu, V. Philipps, S. Brezinsek, M. Lehnen, P. Coad, C. Grisolia, K. Schmid, K. Krieger, A. Kallenbach, B. Lipschultz, R. Doerner, R. Causey, V. Alimov, W. Shu, O. Ogorodnikova, A. Kirschner, G. Federici, and A. Kukushkin, J. Nucl. Mater. 390-391, 1 (2009).

${ }^{2}$ P. Goldstrass and Ch. Linsmeier, Nucl. Instrum. Methods Phys. Res. B 161-163, 411 (2000).

${ }^{3}$ F. Kost, Ch. Linsmeier, M. Oberkofler, M. Reinelt, M. Balden, A. Herrmann, and S. Lindig, J. Nucl. Mater. 390-391, 975 (2009).

${ }^{4}$ A. Anghel, C. Porosnicu, C. P. Lungu, K. Sugiyama, C. Kreiger, and J. Roth, J. Nucl. Mater. 416, 9 (2011).

${ }^{5}$ R. A. Pitts, A. Kukushkin, A. Loarte, A. Martin, M. Merola, C. E. Kessel, V. Komarov, and M. Shimada, Phys. Scr. T138, 014001 (2009).

${ }^{6}$ D. Nishijima, M. J. Baldwin, R. P. Doerner, and R. Seraydarian, J. Nucl. Mater. 363-365, 1261 (2007).

${ }^{7}$ M. J. Baldwin and R. P. Doerner, Nucl. Fusion 46, 444 (2006).

${ }^{8}$ M. J. Baldwin, R. P. Doerner, D. Nishijima, K. Schmid, D. G. Whyte, J. G. Kuplin, and G. Wright, J. Nucl. Mater. 358, 96 (2006).

${ }^{9}$ C. Björkas, N. Juslin, H. Timko, K. Vörtler, K. Nordlund, K. Henriksson, and P. Erhart, J. Phys.: Condens. Matter 21, 445002 (2009).

${ }^{10}$ M. Mehine, C. Björkas, K. Vörter, K. Nordlund, and M. I. Airila, J. Nucl. Mater. 414, 1 (2011).

${ }^{11}$ D. Borodin, A. Kirschner, S. Droste, R. Doerner, D. Nishijima, M. Baldwin, A. Pigarov, E. Hollmann, Gh. Antar, R. Serayderian, V. Philipps, Ph. Mertens, and U. Samm, Phys. Scr. T128, 127 (2007).

${ }^{12}$ A. Kirschner, D. Borodin, S. Droste, V. Philipps, U. Samm, G. Federici, A. Kukushkin, and A. Loarte, J. Nucl. Mater. 363-365, 91 (2007).

${ }^{13}$ Y. Ferro, N. Fernandez, A. Allouche, and Ch. Linsmeier, J. Phys.: Condens. Matter 25, 15002 (2013).

${ }^{14}$ X. He, Z. X. Chen, Z. Li, and Z. Zou, J. Chem. Phys. 133, 231104 (2010).

${ }^{15}$ S. M. Choi and S. H. Jhi, Phys. Rev. Lett. 101, 266105 (2008).

${ }^{16}$ P. Giannozzi, S. Baroni, N. Bonini, M. Calandra, R. Car, C. Cavazzoni, D. Ceresoli, G. L. Chiarotti, M. Cococcioni, I. Dabo, A. Dal Corso, S. de Gironcoli, S. Fabris, G. Fratesi, R. Gebauer, U. Gerstmann, C. Gougoussis, A. Kokalj, M. Lazzeri, L. Martin-Samos, N. Marzari, F. Mauri, R. Mazzarello, S. Paolini, A. Pasquarello, L. Paulatto, C. Sbraccia, S. Scandolo, G. Sclauzero, A. P. Seitsonen, A. Smogunov, P. Umari, and R. M. Wentzcovitch, J. Phys.: Condens. Matter 21, 395502 (2009).

${ }^{17}$ D. Vanderbilt, Phys. Rev. B 41, 7892 (1990).

${ }^{18}$ J. P. Perdew, K. Burke, and M. Ernzerhof, Phys. Rev. Lett. 77, 3865 (1996).

${ }^{19}$ V. Barone, M. Casarin, D. Forrer, M. Pavone, M. Sambi, and A. Vittadini, J. Comput. Chem. 30, 934 (2009).

${ }^{20}$ S. Grimme J. Comput. Chem. 27, 1787 (2006).

${ }^{21}$ G. Henkelman, B. P. Uberuaga, and H. Jonsson, J. Chem. Phys. 113, 9901 (2000).

${ }^{22}$ G. Henkelman and H. Jonsson, J. Chem. Phys. 113, 9978 (2000).

${ }^{23}$ Y. Ferro and A. Allouche, Phys. Rev. B 75, 155438 (2007).

${ }^{24}$ Y. Ferro, D. Teillet-Billy, N. Rougeau, V. Sidis, S. Morisset, and A. Allouche, Phys. Rev. B 78, 85417 (2008).

${ }^{25}$ H. J. Monkhorst and J. D. Pack, Phys. Rev. B 13, 5188 (1976). 
${ }^{26}$ S. Evangelisti, A. Monari, T. Leninger, and G. L. Bendazzoli, Chem. Phys. Lett. 496, 306 (2010).

${ }^{27}$ V. Vetere, A. Monari, A. Scemama, G. L. Bendazzoli, and S. Evangelisti, J. Chem. Phys. 130, 024301 (2009).

${ }^{28}$ J. S. Bunch, S. S. Verbridge, J. S. Alden, A. M. van der Zande, J. M. Parpia, H. G. Craighead, and P. L. McEuen, Nano Lett. 8, 2458 (2008).

${ }^{29}$ O. Leenaerts, B. Partoens, and F. M. Peeters, Appl. Phys. Lett. 93, 193107 (2008).

${ }^{30}$ Y. Ferro, F. Marinelli, and A. Allouche, J. Chem. Phys. 116, 8124 (2002).

${ }^{31}$ H. Amara, S. Latil, M. Meunier, Ph. Lambin, and J. C. Charlier, Phys. Rev. B 76, 115423 (2007).

${ }^{32}$ P. O. Lethinen, A. S. Foster, Y. Ma, A. V. Krasheninnikov, and R. M. Nieminen, Phys. Rev. Lett. 93, 187202 (2004).

${ }^{33}$ Z. He, X. Yang, H. Xia, T. Z. Regier, D. K. Chevrier, X. Zhou, and T. K. Sham, Phys. Rev. B. 85, 144406 (2012).

${ }^{34}$ V. M. Pereira, F. Guinea, J. M. B. Lopes dos Santos, N. M. R. Peres, and A. H. Castro Neto, Phys. Rev. Lett. 96, 36801 (2006).

${ }^{35}$ A. Allouche and Y. Ferro, Carbon 44, 3320 (2006).

${ }^{36}$ Y. Ferro, F. Marinelli, A. Allouche, and C. Brosset, J. Nucl. Mater. 321, 294 (2003).

${ }^{37}$ Y. Ferro, F. Marinelli, A. Allouche, and C. Brosset, Surf. Sci. 559, 158 (2004).
${ }^{38}$ M. Fujita, K. Wakabayashi, K. Nakada, and K. Kusakabe, J. Phys. Soc. Jpn. 65, 1920 (1996).

${ }^{39}$ K. Nakada, M. Fujita, G. Dresselhaus, and M. Dresselhaus, Phys. Rev. B 54, 17954 (1996).

${ }^{40}$ Y. Miyamoto, K. Nakada, and M. Fujita, Phys. Rev. B 59, 9858 (1999).

${ }^{41}$ Y. Niimi, T. Matsui, H. Kambara, K. Tagami, M. Tsukada, and H. Fukuyama, Phys. Rev. B 73, 85421 (2006).

${ }^{42}$ Y. Kobayashi, K. Fukui, T. Enoki, and K. Kusakabe, Phys. Rev. B 73, 125415 (2006).

${ }^{43}$ Y. Kobayashi, K. Fukui, T. Enoki, K. Kusakabe, and Y. Kaburagi, Phys. Rev. B 71, 193406 (2005).

${ }^{44}$ Y. Son, M. L. Cohen, and S. G. Louie, Nature 444, 347 (2006).

${ }^{45}$ J. Kuntsmann, C. Ödogan, A. Quandt, and H. Fehske, Phys. Rev. B 83, 45414 (2011).

${ }^{46}$ H. Lee, Y. Son, S. Han, and J. Yu, Chem. Phys. Lett. 398, 207 (2004).

${ }^{47}$ N. Emery, C. Hérold, and P. Lagrange, J. Phys. Chem. Solids 67, 1137 (2006).

${ }^{48}$ N. Emery, C. Hérold, and P. Lagrange, Solid State Chem. 178, 2947 (2005).

${ }^{49}$ C. Ataca, E. Aktürk, and S. Ciraci, Phys. Rev. B 79, 41406(R) (2009).

${ }^{50}$ G. Srinivas, C. A. Howard, S. M. Bennington, N. T. Skipper, and M. Ellerby, J. Mater. Chem. 19, 5239 (2009).

${ }^{51}$ M. Cobian and J. Iniguez, J. Phys.: Condens. Matter 20, 285212 (2008). 